5
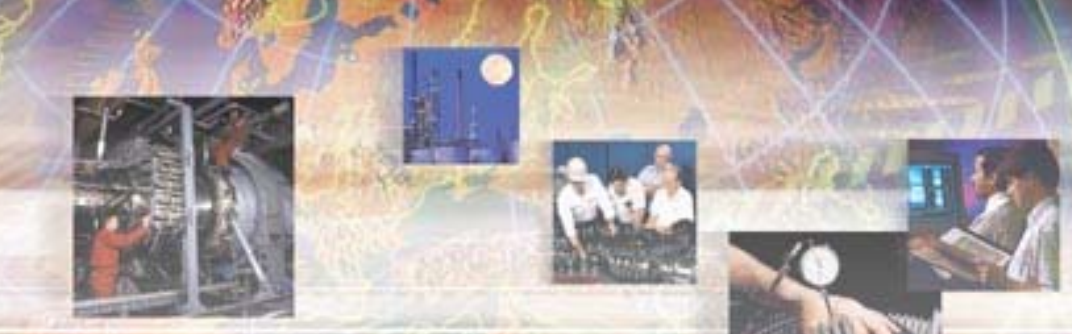

\title{
Diagnosis and Solution of High Noise and Vibration Issues After a Propylene Refrigeration Compressor Re-rate
}

\author{
$B y$ \\ Zheji Liu \\ Mark J. Kuzdzal \\ Dresser-Rand \\ Kenneth E. Atkins \\ Engineering Dynamics Inc. \\ Randy Rials \\ Westlake Chemical
}




\section{- Introduction}

- Noise is a working environment concern

- excessive noise exposure can cause hearing damage, raising stress level, and interfere with verbal communication.

- Excessive noise and vibration may inflict fatigue damage on mechanical systems

- This is the focus of this study. 


\section{History}

- A propylene refrigeration turbo-compressor installed in a Chemical Plant was revamped in 2006 to increase the volumetric flow rate with new stationary and rotating components.

- In October 2006, the centrifugal compressor was restarted and significant noise and piping vibration was observed from the discharge pipe attached to the $4^{\text {th }}$ nozzle.

- The level of noise and vibration appeared to vary slightly with the flow rate, but even at relatively low flow rates the vibration and noise was excessive.

- The discharge piping downstream of nozzle 4 had some vibration since startup in 1998, but was nowhere near the levels that were now being observed. 


\section{Description of the Process Compressor}

- The subject compressor draws gas

from two process pipes and

discharges gas through three

discharge pipes.

- Main inlet 1: 42-inch-diameter .

- 2nd inlet: a 20-inch nozzle (2) to bring in additional gas.

- 1st outlet: a 10-inch nozzle (3) to take gas out of compressor.

- 2nd outlet: an 8-inch nozzle (4) (This impeller/nozzle/discharge pipe is the focus of this study). The 8-inch nozzle is then transitioned into a 12-inchdiameter discharge pipe (4D).

- Final discharge: 20-inch-diameter final discharge nozzle (5).

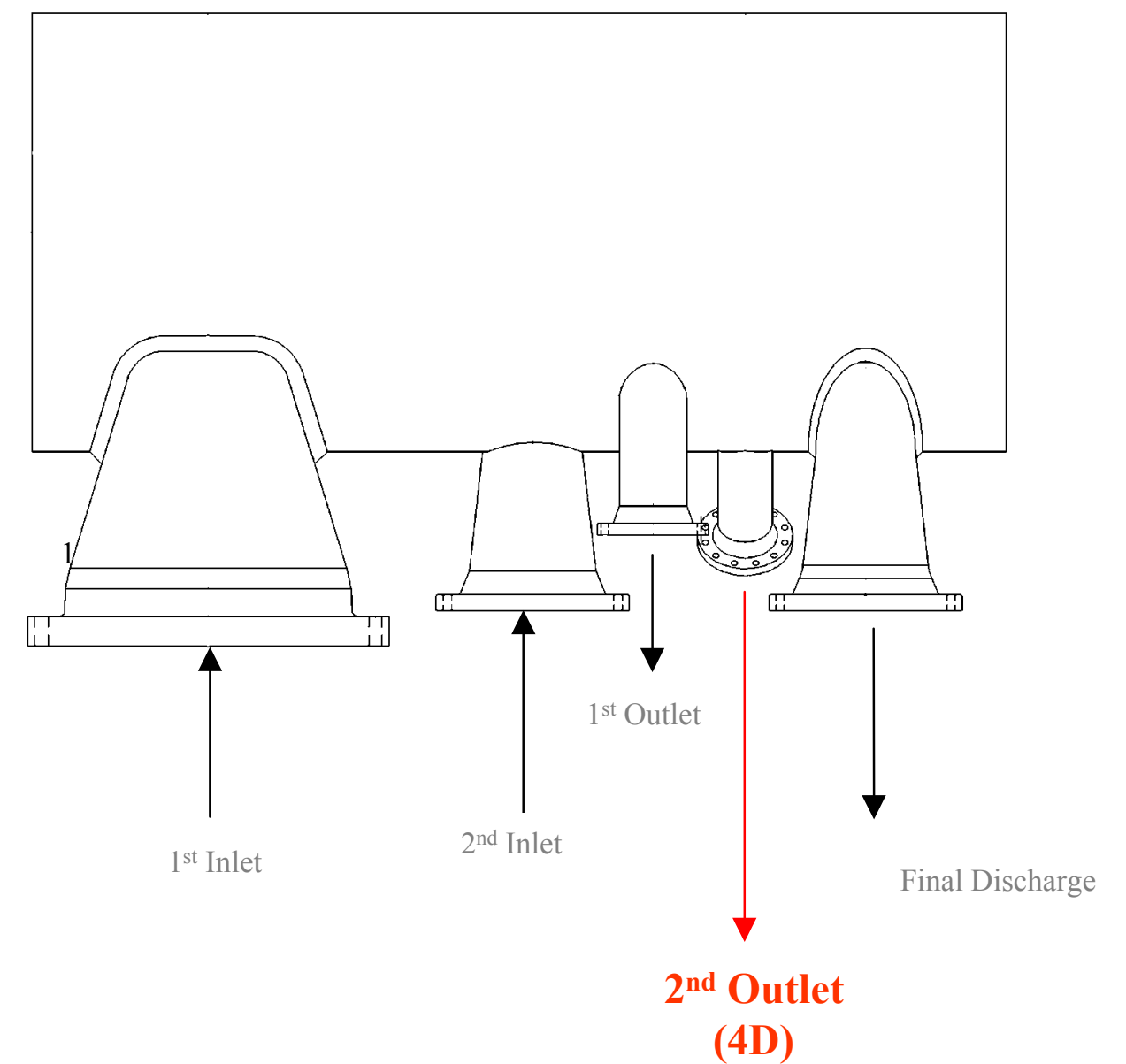




\section{Noise and Vibration Issue}

- High noise and

vibration was

observed only on the

4D pipe.

- It caused external piping connections on the 4D pipe to develop cracks.

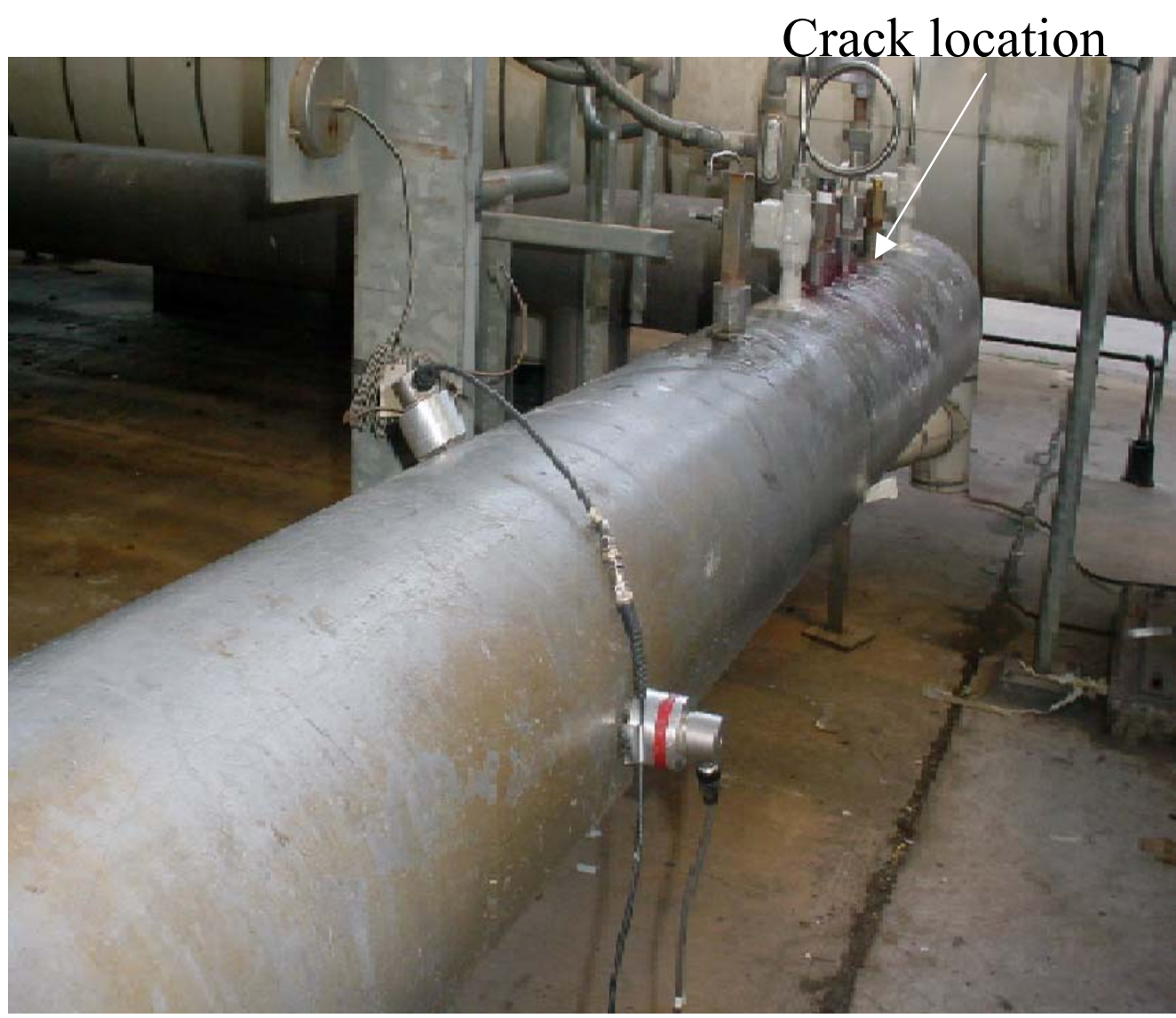




\section{-First Attempt}

- Plant engineering resources initially contacted an outside firm to assist in determining the cause of the high levels of vibration and noise.

- Pressure pulsations, piping vibration and dynamic strain were measured on the 4D pipe.

- The conclusion from the first consultant was that the "Mokveld" check valve located downstream of the compressor was inducing an "acoustic" vibration resonance on the piping. Vortex shedding was claimed as the root cause of the high noise and vibration.

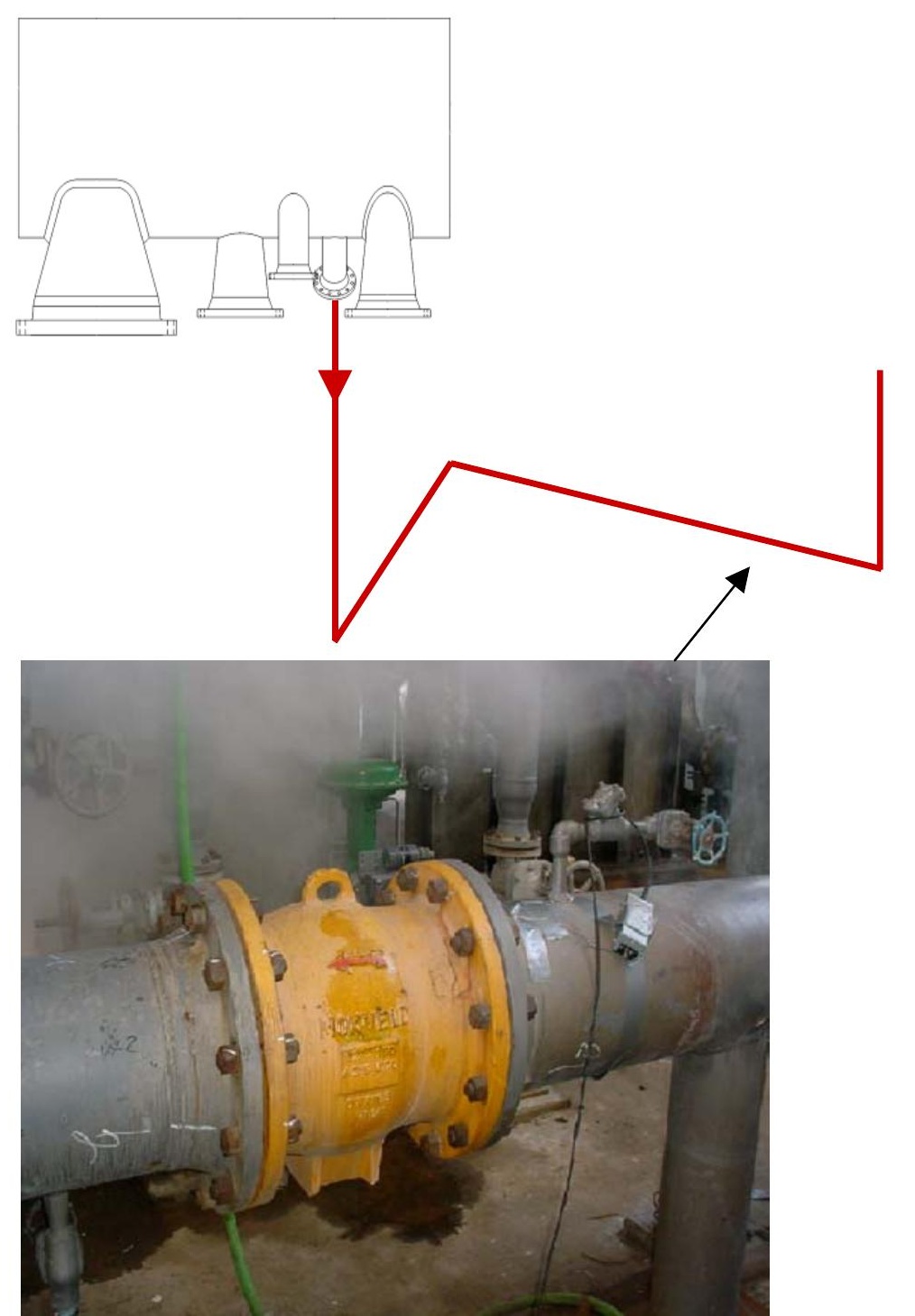




\section{- Noise and Vibration Remain High}

- In December 2006, the production unit was shutdown and a new "Wafer" check valve and replacement piping was installed.

- Upon restart of the propylene compressor, the piping vibration and noise levels returned to or near the same levels as before.

- The investigation into the high noise and vibration in the discharge piping downstream of nozzle 4 continued with another troubleshooting test on January 12, 2007. This test program was undertaken by the authors of this study. 


\section{Root Cause Diagnosis}

- Pressure pulsations, piping vibration, dynamic strain, and sound pressure level were measured on the 4D pipe.

- Sound Intensity level was measured along 4D pipe. (Additional)

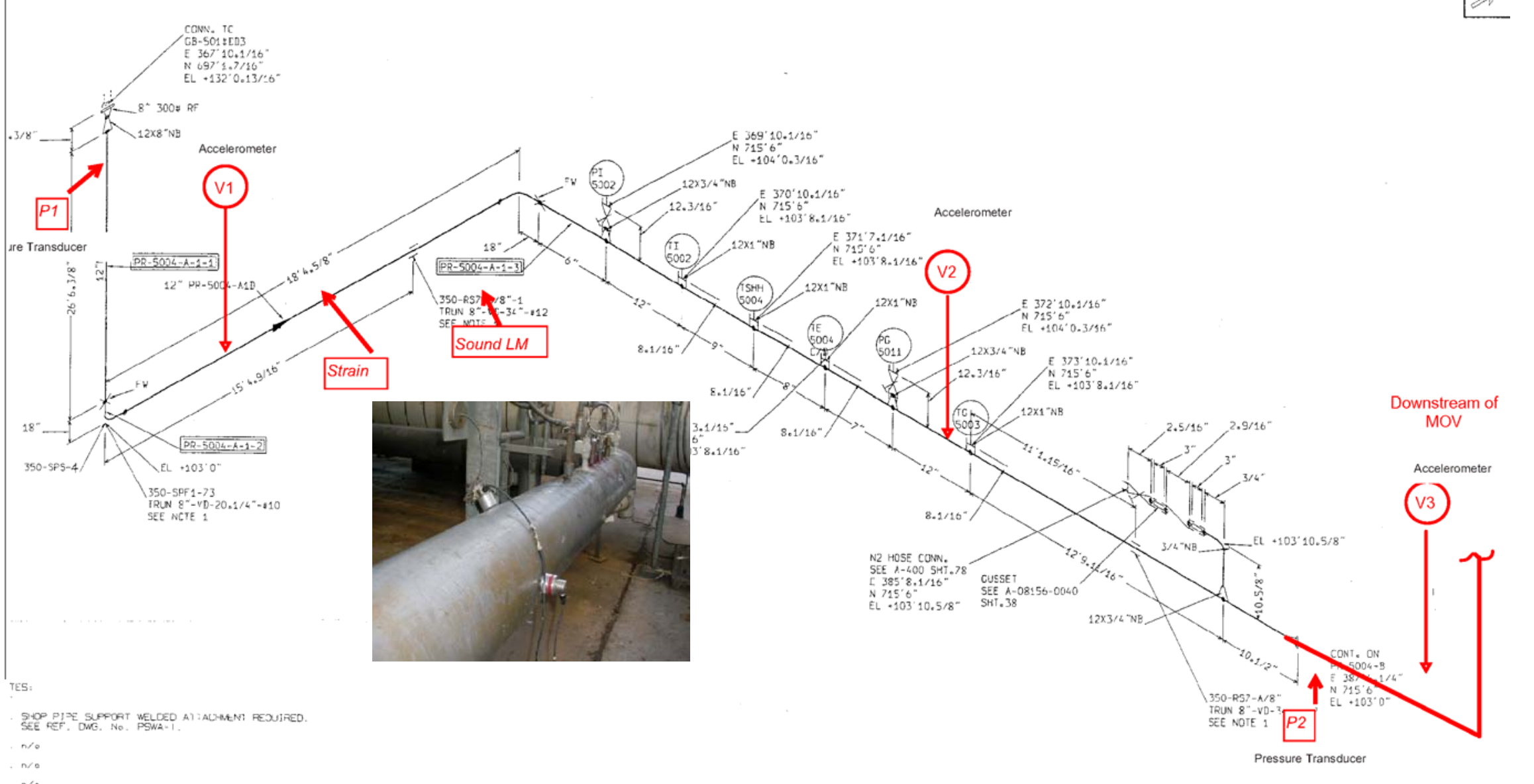




\section{Test Conditions}

- Test lasted about 9

hours, dynamic

signals (pulsation, vibration, strain and noise) were continuously recorded.

- Speed, flow rate, pressure, and temperature were varied during the test.

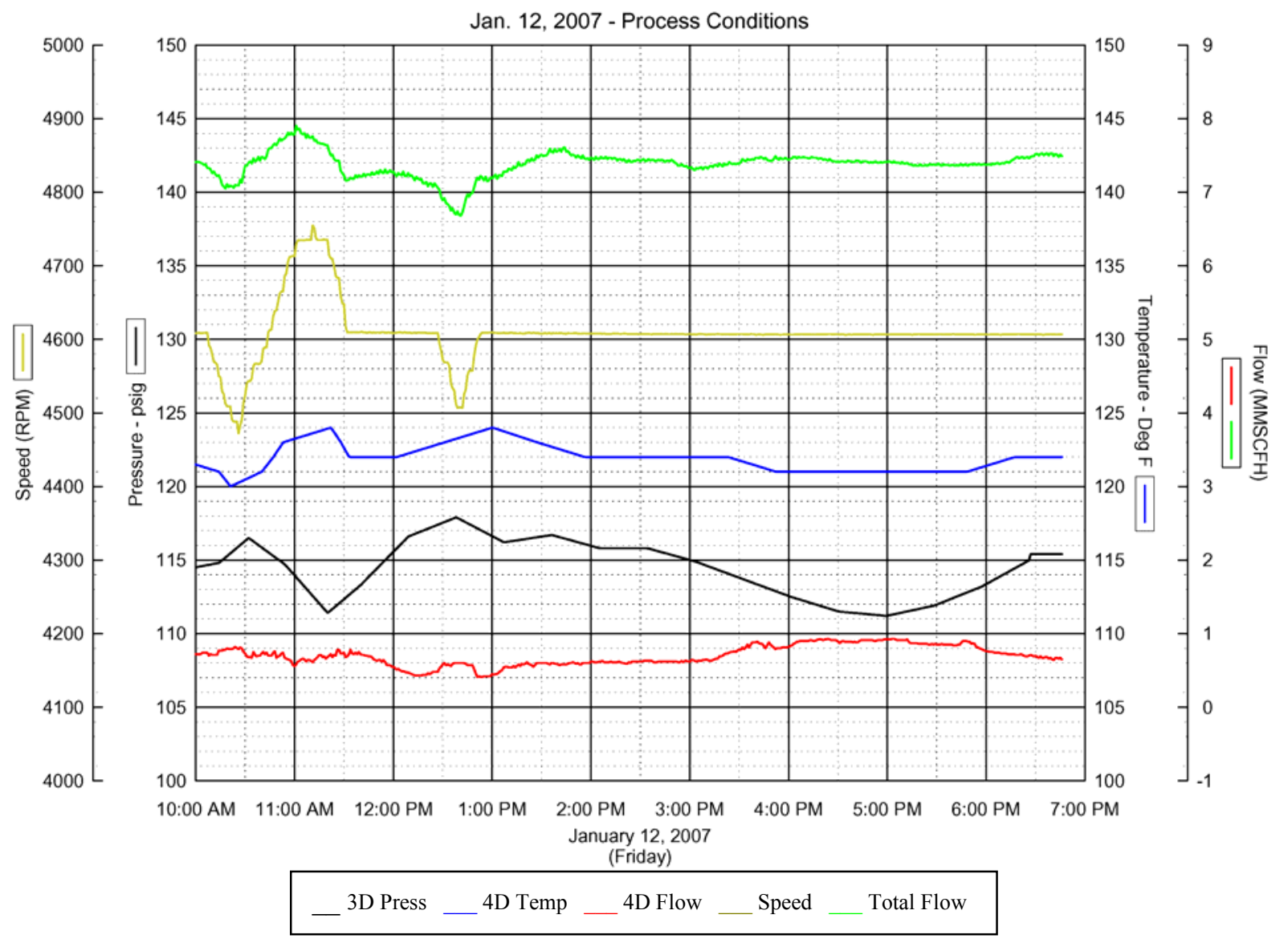




\section{Source Identification}

- by Frequency Based Technique

- Both sound pressure level and sound intensity level were acquired in narrow band (FFT).

- Noise source is discrete in nature with strong tonal components - fundamental at $480 \mathrm{~Hz}$, and integer multiples at $960 \mathrm{~Hz}$ and $1440 \mathrm{~Hz}$.

- This type of noise signature is not likely from a Vortex shedding phenomenon.

- It can not be a sole result of either acoustical or structural resonance of the 4D pipe.

- The excitation source is not likely within the 4D pipe.
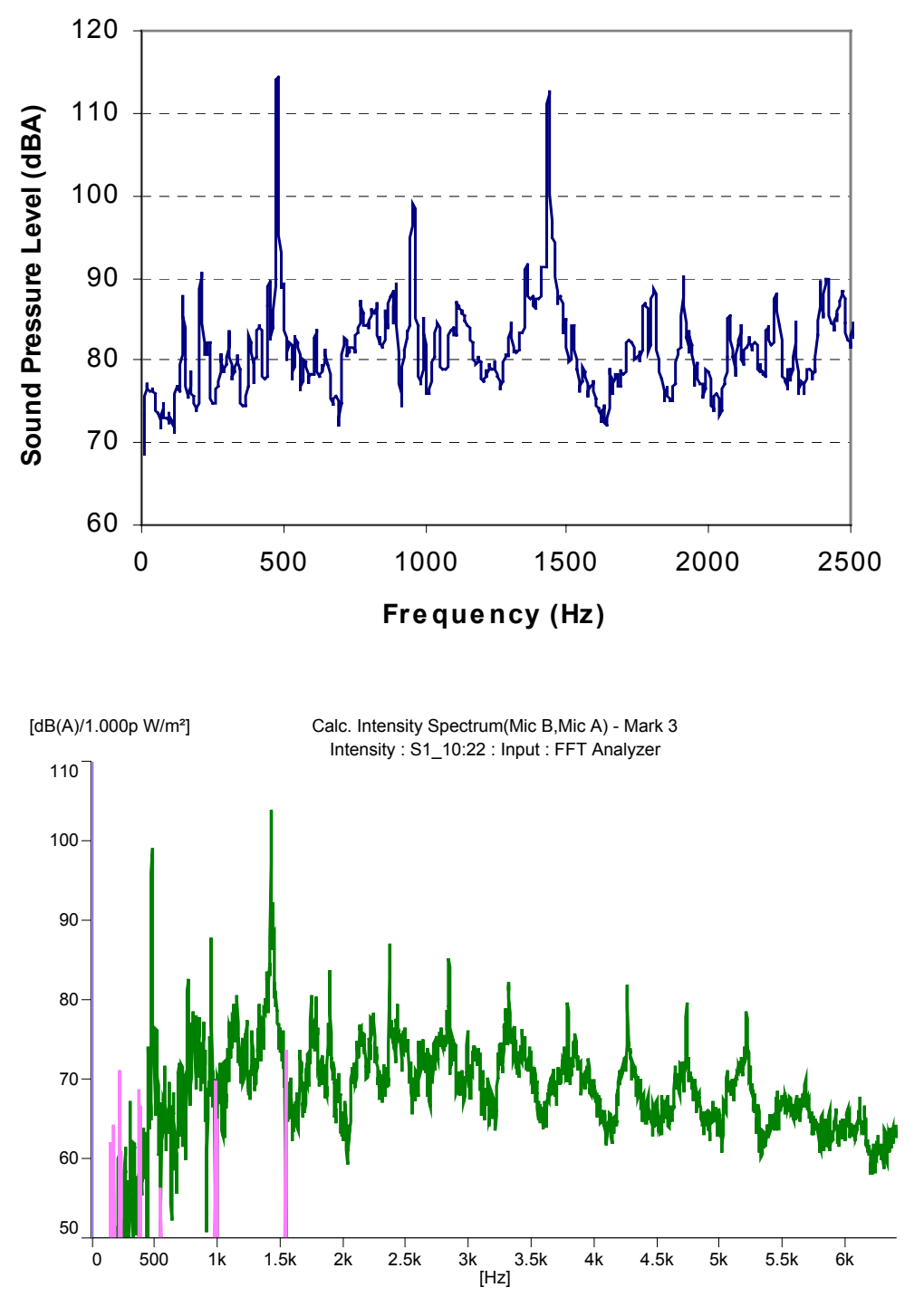


\section{Source Identification - by Frequency (continued)}

- Pulsations, vibration, and dynamic strain data show strong coherence among them.

- All have peaks at $480 \mathrm{~Hz}$ (fundamental); 960 $\mathrm{Hz}$ (2nd harmonic), and 1440 (3rd harmonics).

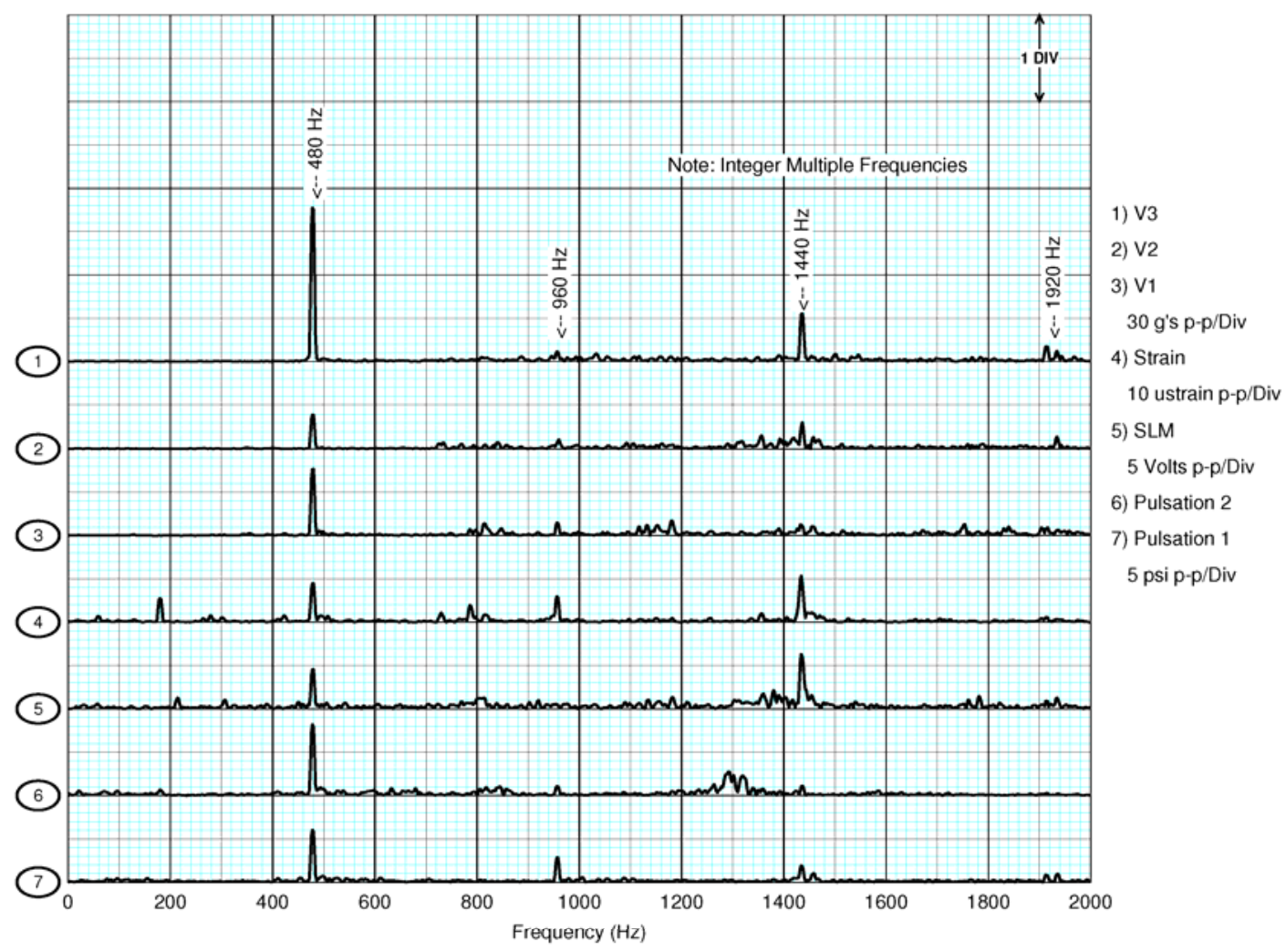




\section{Source identification - by Spatially Based Technique (Sound Intensity)}

- Measuring noise spatial variation, sound intensity scanning is an excellent technique for noise source identification.

- Uniform intensity levels along the 4D pipe indicates the excitation source is from upstream of the 4D pipe the compressor.

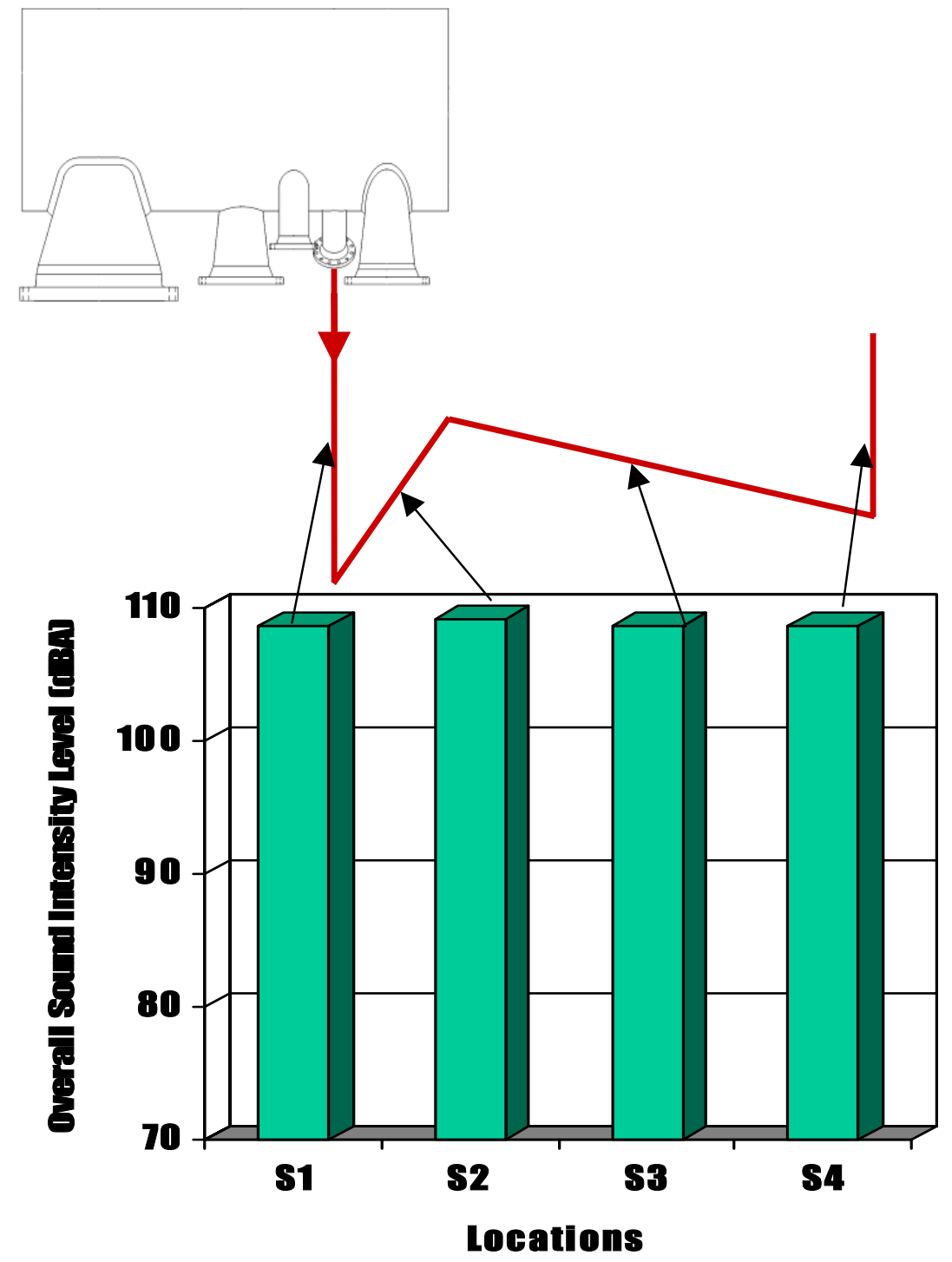




\section{Source Identification - by Condition Variations}

- Peak pulsation frequency does not track to speed - not a rotational source.

- It also does not correlate to flow rate - not a vortex shedding

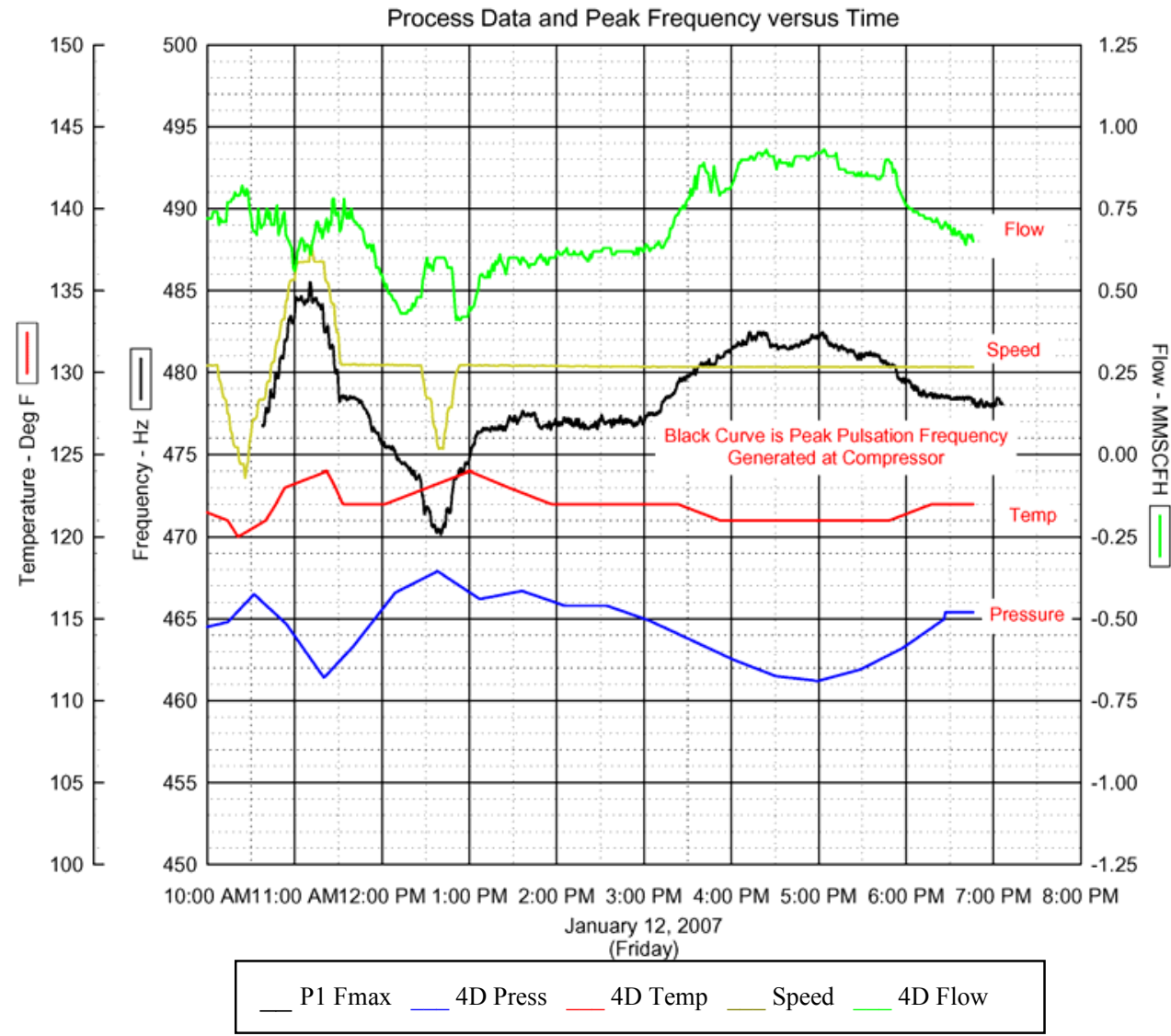
source. 


\section{Root Cause and Solution}

- The source of the high noise and vibration on the 4D pipe was successfully diagnosed with various noise source identification techniques

- Frequencies - already known; strong discrete tones at 480 , and $1440 \mathrm{~Hz}$.

- Source Location - not the 4D pipe but the compressor.

- Further analysis of the geometry in the side stream volute upstream of the 4D discharge piping lead to the belief that standing waves in the modified collector were the root cause of the high noise and vibration.

- With the excitation source correctly identified, a solution of using duct resonator array was prescribed to the Propylene compressor.

- This provides quick turnaround time than modifying machine internal.

- The excitation is not harmful to compressor due to poor coupling.

- Duct resonator array is a proven noise control technology that has been successfully implemented to 170 compressors.

- Typically reduce noise level by $10+\mathrm{dBA}$ and vibration level by $90 \%$. 


\section{Duct Resonator Array - Internal}

- Using Duct Resonator array to reduce the compressor noise source internally by lowering

- Pressure Pulsations

- Dynamic Excitations

- Structural Vibration

- Noise Radiation.

- Benefits include

- Reduce unsteady aerodynamic load on impeller blades and diffuser vanes.

- Reduce piping vibration and improve structure integrity and instrumentation life.

- Reduce compressor noise level.

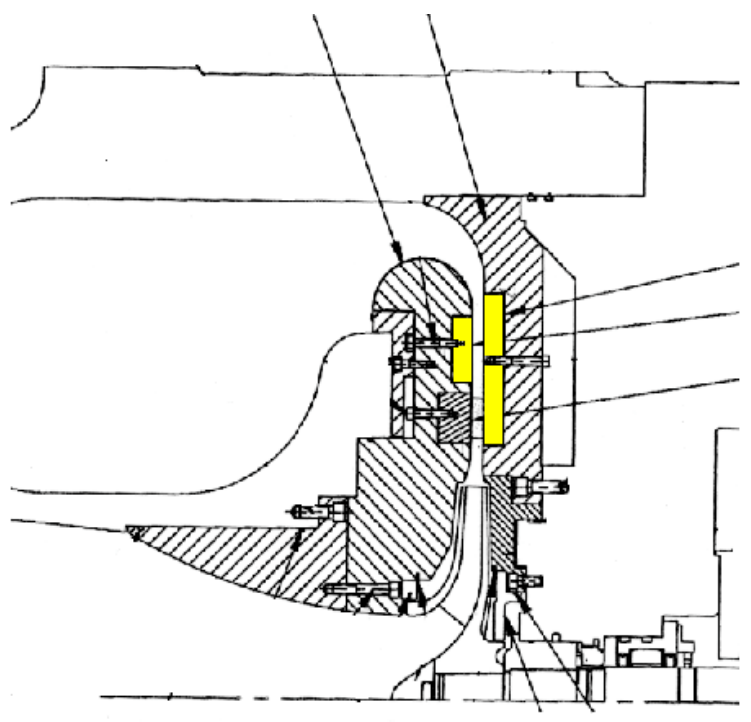

Require machine internal modification 


\section{Pipe DR Array - External}

- Applying the duct resonator array technology to the compressor inlet and discharge pipe.

- Lining the inner wall of the inlet/discharge pipe (spool piece) with duct resonator array.

- No need to disassemble compressor.

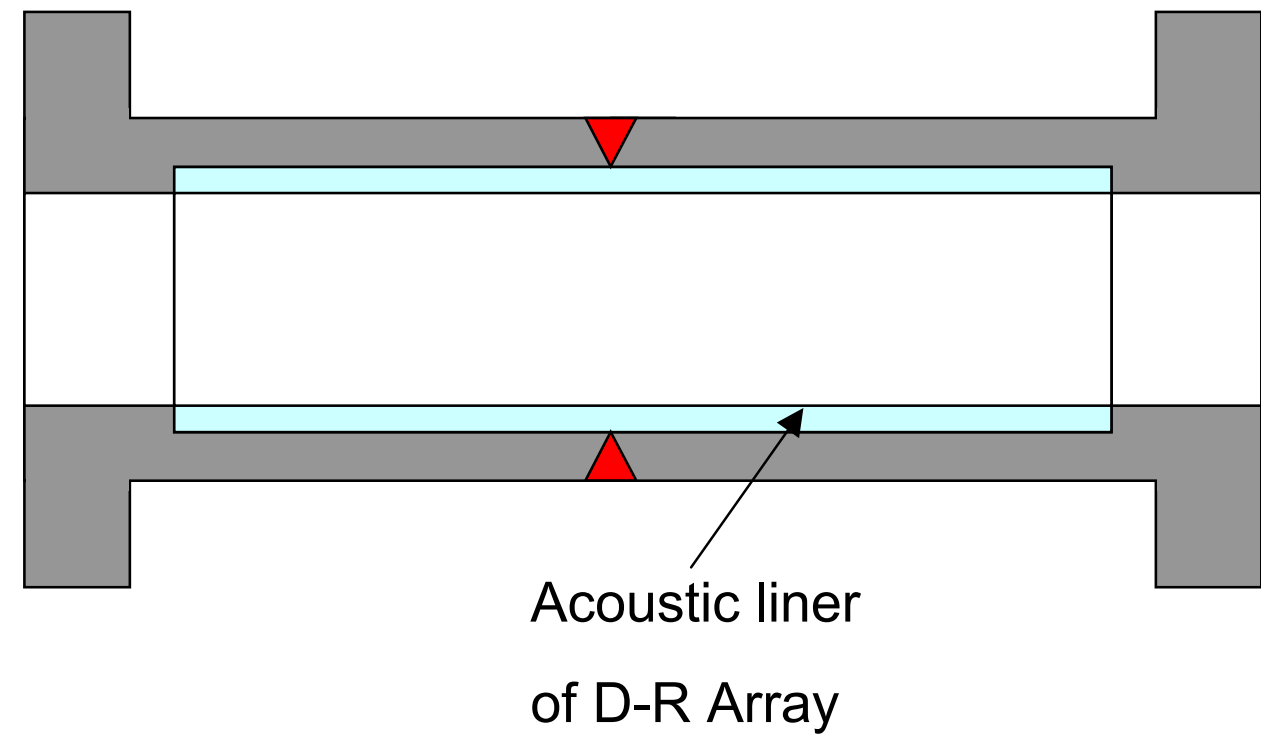




\section{Two DoF Pipe Array}

- A two DoF pipe array was designed to reduce noise and vibration at $480 \mathrm{~Hz}$ and $1440 \mathrm{~Hz}$.

- It requires no compressor internal modification and provides quick turnaround.

- As it is mounted on the 4D nozzle, the pipe array minimizes the excitation energy entering the 4D pipe.

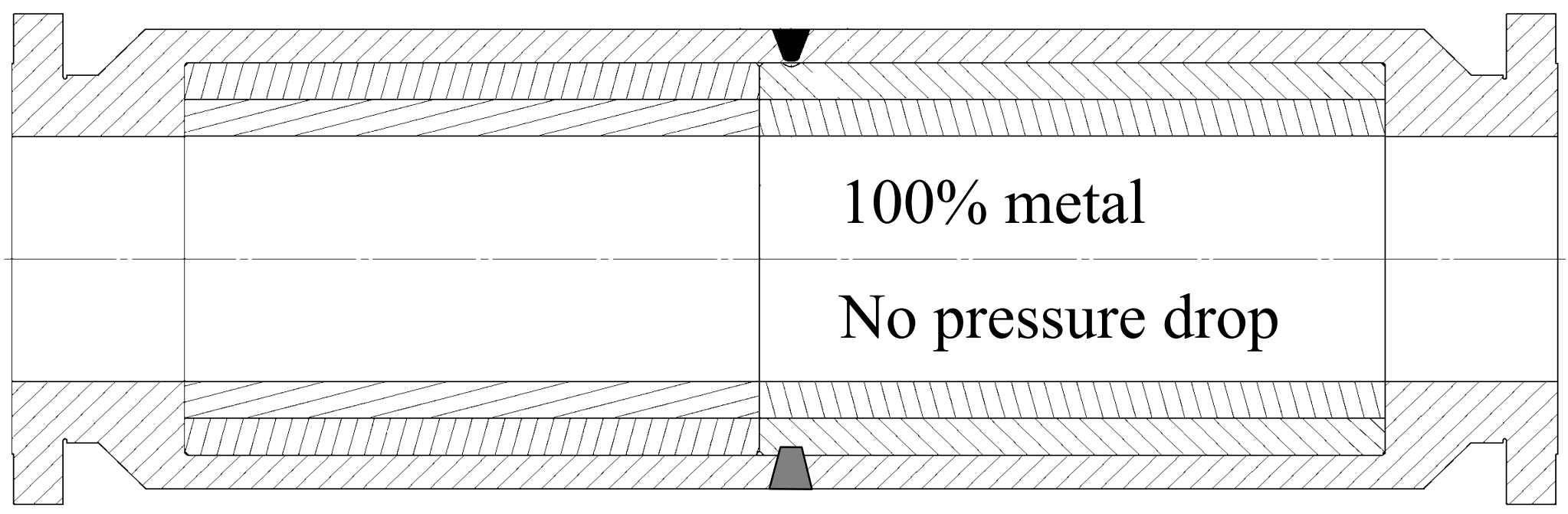




\section{- Impedance Tube Test}

- An impedance tube with an acoustic driver was used to test the pipe array via a transition cone.

- Microphones positioned upstream and downstream of the array to measure the sound pressure level difference across the array.

- A PC driven B\&K Pulse Front End was used for

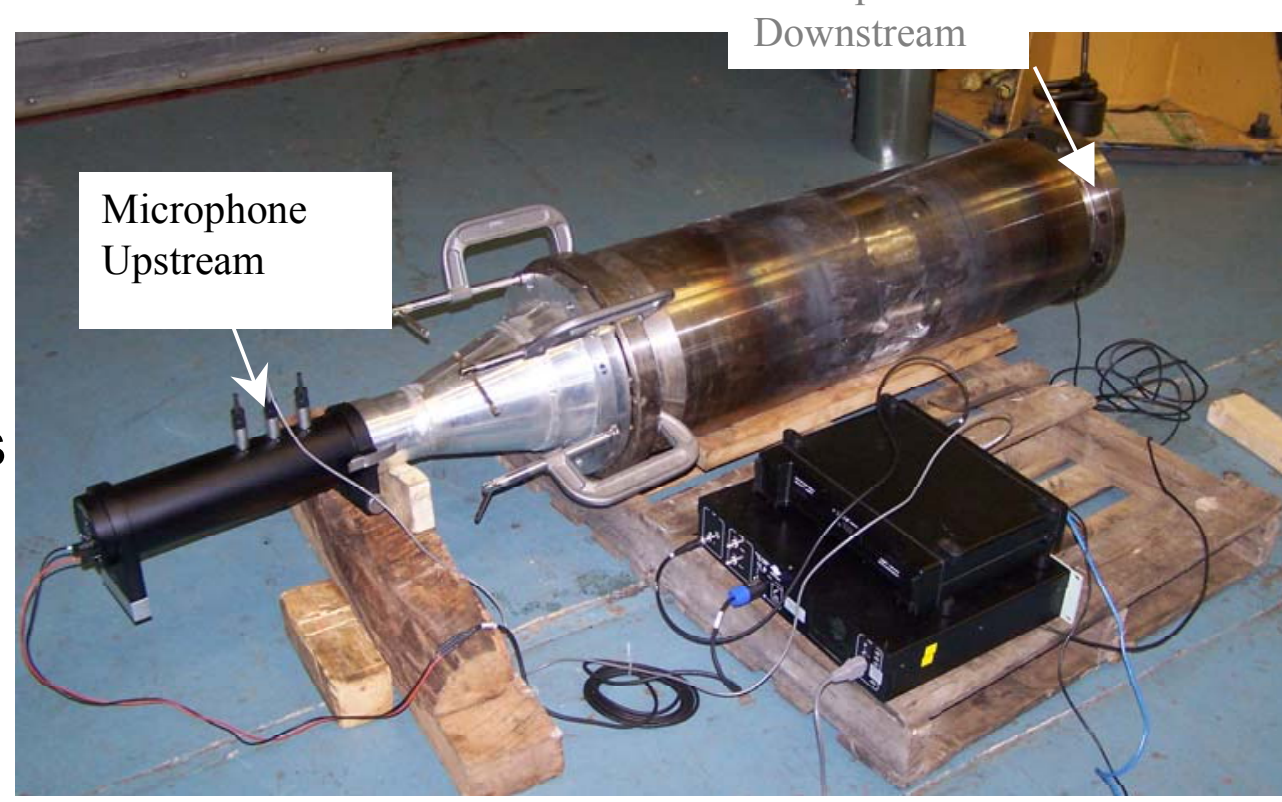

- Generating noise signal

- Measuring noise 


\section{Sound Pressure Levels Comparison}

- Discrete noise source with tones at $700 \mathrm{~Hz}$ and $1900 \mathrm{~Hz}$ was applied.

- This is to emulate the $480 \mathrm{~Hz}$ and $1440 \mathrm{~Hz}$ dominant noise components at site.

- This frequency shift is due to change in sonic velocity of the gas (Propylene vs. Air).

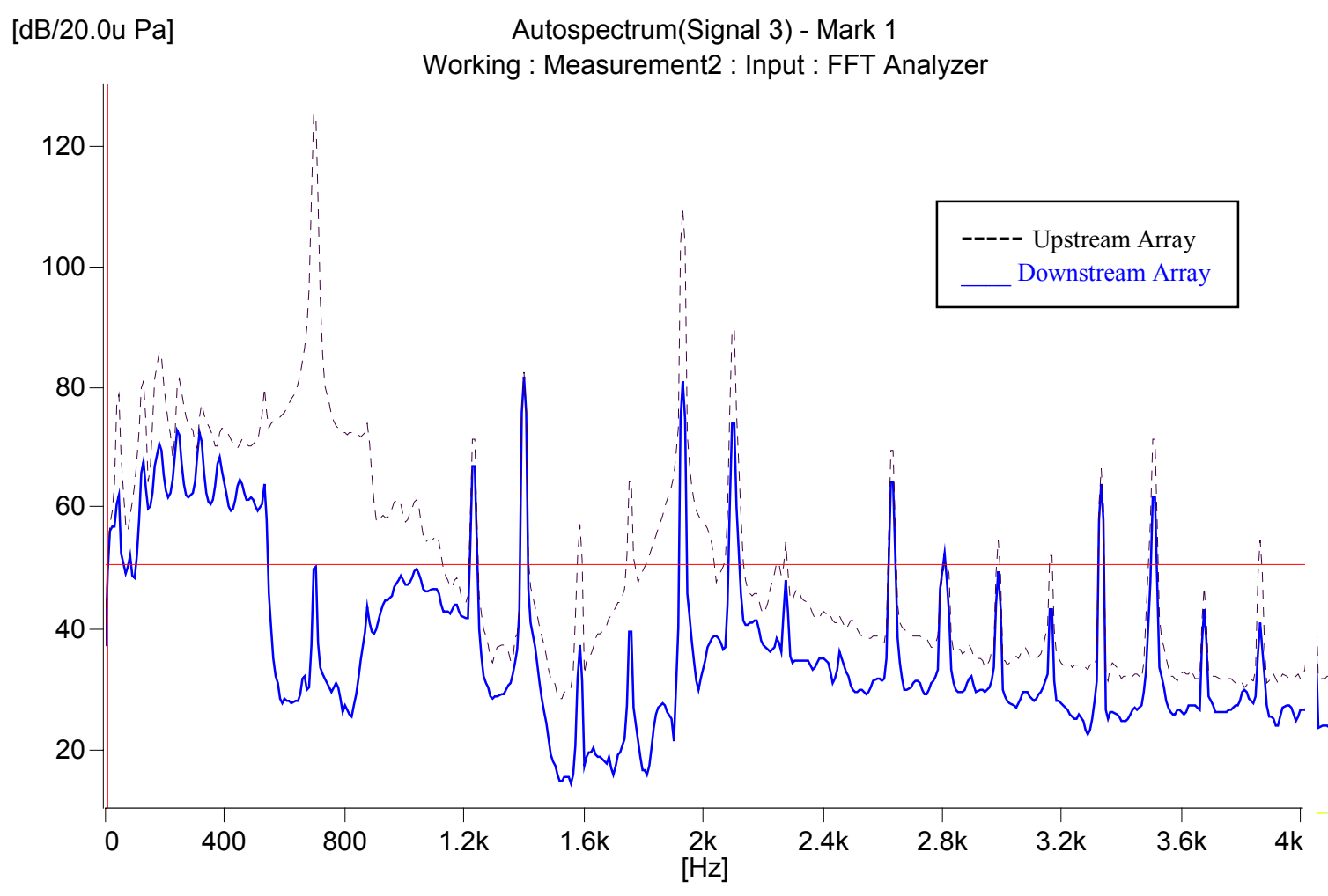




\section{Sound Pressure Levels Comparison (continued)}

- Broadband noise source was also fed into the pipe array.

$\rightarrow$ This comparison demonstrates the noise attenuation bandwidth(s) of the array.

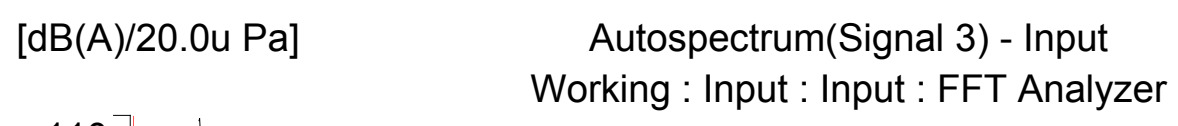

[dB(A)/20.0u Pa]

Autospectrum(Signal 3) - Input
Working : Input : Input : FFT Analyzer

110

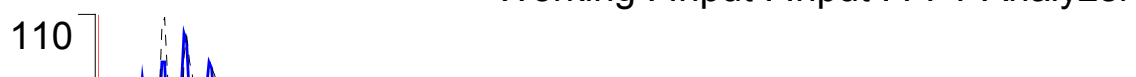

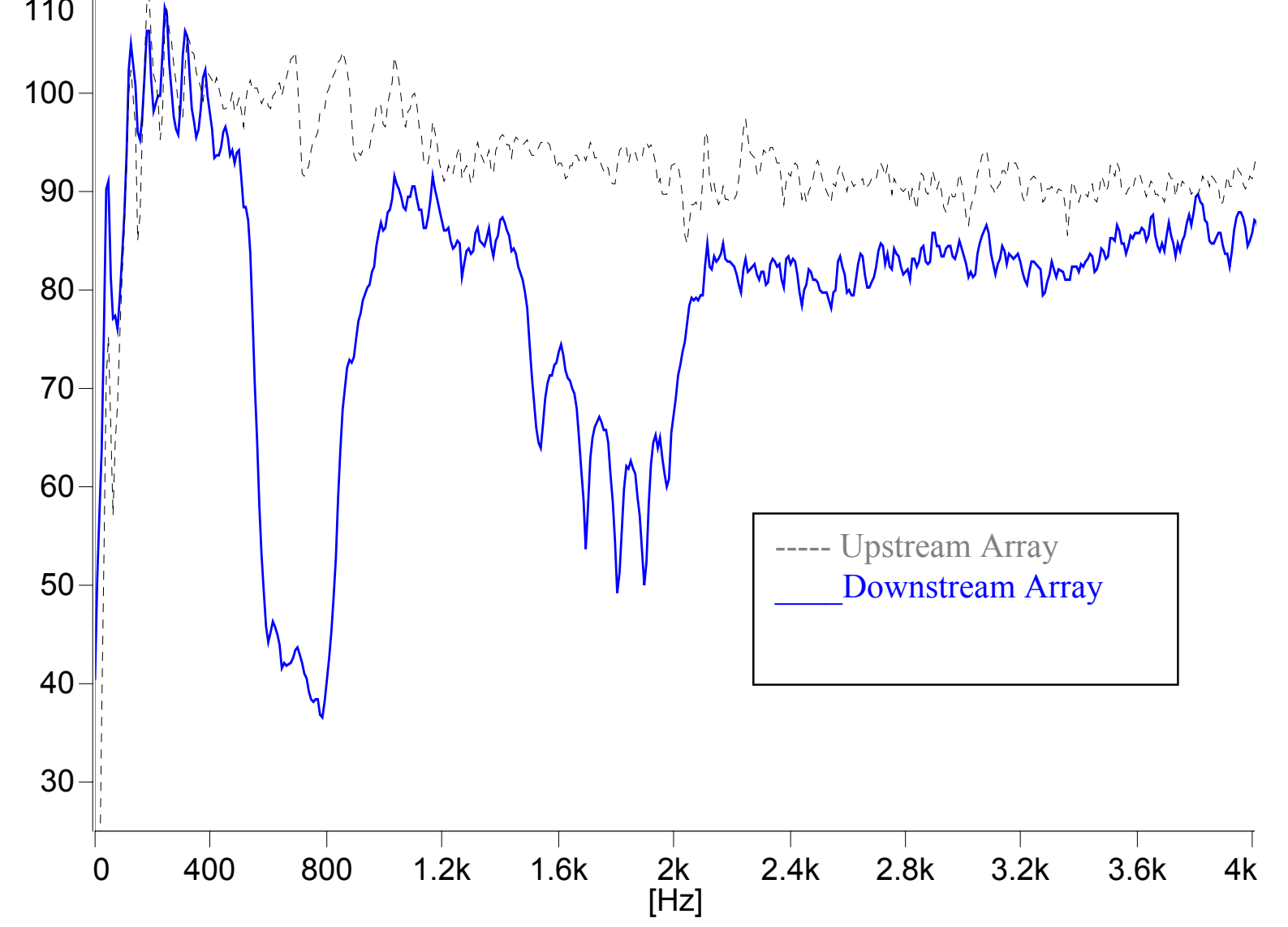




\section{-Final Test}

- After the pipe array was inserted between the compressor $2^{\text {nd }}$ outlet (nozzle 4) and the 4D discharge pipe, follow-up testing was conducted to verify the results.

- The test procedure was to install pressure transducers upstream and downstream of the pipe array and record pulsation data at process conditions similar to those that resulted in excessive pulsation, vibration, and noise levels during the initial testing in January 2007.
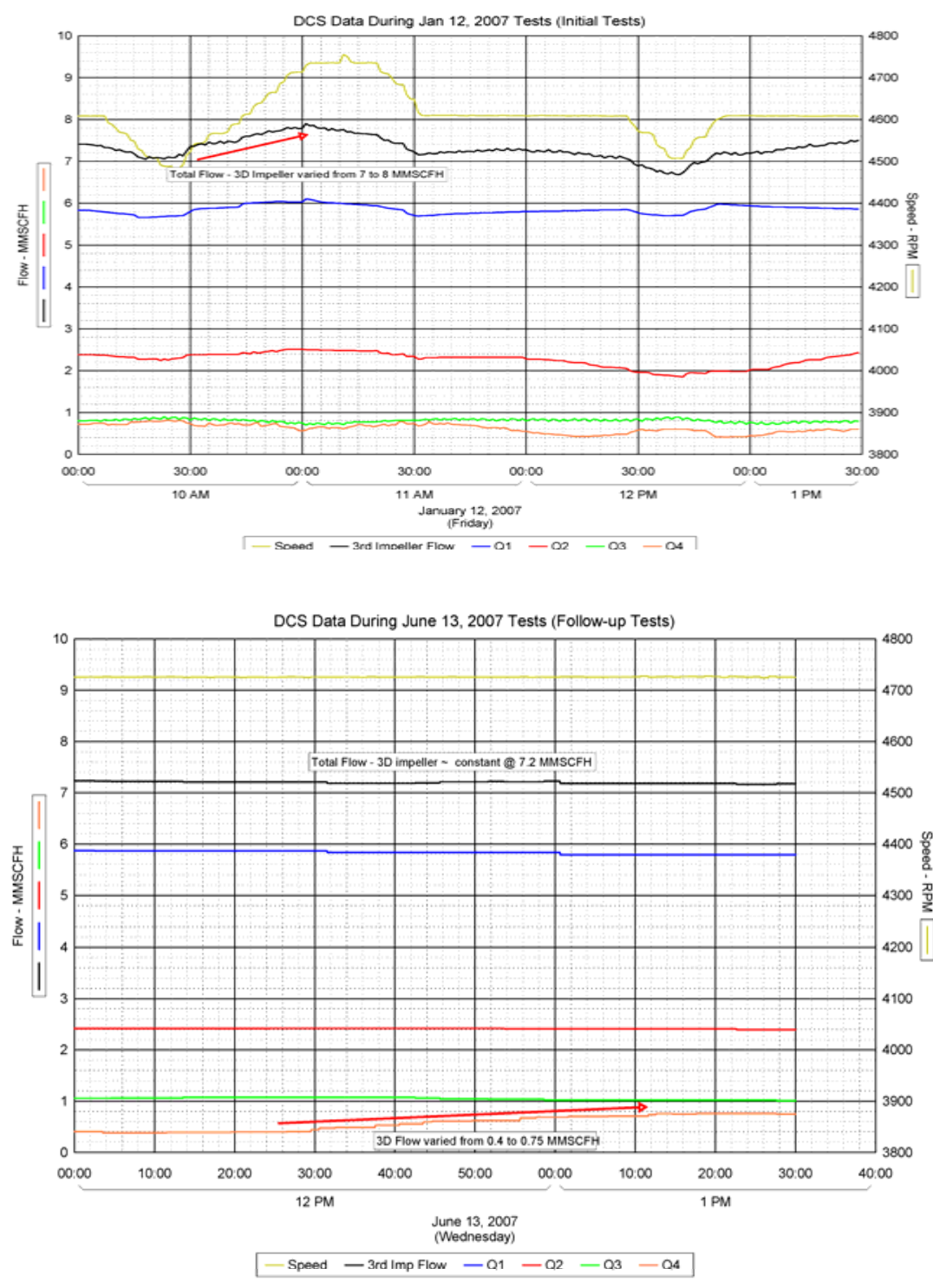


\section{Data Comparison (Array Effect)}

- The final test shows

virtually no pulsation near $480 \mathrm{~Hz}$ (red curve),

whereas this location had about 2.3 psi pk-pk during the initial test on January 12, 2007.

- The pressure pulsation at $1440 \mathrm{~Hz}$ was also completely eliminated by the introduction of the resonator array.

- Adding the pipe array corrected the problem.






\section{Conclusions}

- In-pipe pressure pulsations, piping vibration, pipe wall strain levels, external sound pressure, and pipe surface sound intensity were measured to identify the excitation source.

- Analysis of the measured data indicated that the acoustic source originated within the compressor, upstream of the side stream discharge nozzle.

- With the excitation source correctly identified, a duct resonator pipe array was designed to reduce the acoustic energy entering the side stream piping.

- After the pipe array was installed in the discharge piping, a second site test was performed to confirm the effectiveness of the array. Pressure pulsation data were measured upstream and downstream of the array, which indicated that the pressure peaks at $480 \mathrm{~Hz}$ and $1440 \mathrm{~Hz}$ were both eliminated.

- The noise and vibration issues on the side stream piping system were successfully resolved. Since the duct resonator array was installed in June, 2007, the chemical plant has not had any issue with the 4D discharge pipe. 